\title{
METHOD OF CONCORDANCE IN THE DIAGNOSIS OF STRABISMUS *†
}

\author{
BY \\ JOSEPH I. PASCAL \\ From the Department of Ophthalmology, N.Y. Polyclinic Medical School and \\ Hospital, New York
}

THIS method is practically unknown to American and British ophthalmologists, and is not mentioned in any text-book in the English language, yet it is not new, for it was described some 30 years ago by Marquez $(1942,1952)$.

It is briefly explained in some Spanish and French texts, and is being used at present in both Europe and South America.

The most probable reason why the method has not been described in the English text-books is that it had to be used with a muscle schema which was not easy to remember, to visualize, or to apply. But the method lends itself admirably to the Benzene Ring Muscle Schema as will be shown presently. As to its value, one need not subscribe to the view (Rivas Cherif, 1949) that it is the best method for diagnosing paralytic and spastic strabismus, for it has good and bad points like all other methods. But it should certainly be known and used, on occasion at least, by every ophthalmologist.

The answer to the question whether we need one more method of diagnosing strabismus is best given in the following quotation from Scobee (1952):

I recently had the rather dubious pleasure of seeing a patient with a vertical imbalance who had previously consulted seven quite reputable ophthalmologists. The patient had carefully tabulated the diagnoses and recommendations of all seven. Truth is often stranger than fiction. Of the eight vertically acting muscles, all but the left inferior oblique had been solemnly identified as the offending muscle. The patient was a person of average intelligence and could hardly be blamed for his somewhat cynical attitude. His problem, obviously enough, was how to know whom to believe, and by the time I saw him he was loth to believe anyone. Cases such as the one mentioned are not isolated instances, unfortunately, but are almost everyday occurrences . . .

In view of the above statement, another method of diagnosing strabismus may be worth knowing.

\section{Method and Apparatus}

No extensive or expensive equipment is required. The only apparatus is a pair of goggles with one red and one green filter (to know which image belongs to which eye), a small, round light (5 or $10 \mathrm{~mm}$.) to serve as target for the first four steps of the examination, and a linear light " target" (12 to $15 \mathrm{~cm}$. long), preferably in the form of an arrow, for the last step in the examination.

The method is essentially a co-ordination of five diplopia tests, the findings of which are grouped on a muscle schema. The letters $\mathbf{P}$ and $\mathrm{S}$, and a dot $(\cdot)$ are used

*Presented before the N.Y. Society for Clinical Ophthalmology, New York Academy of Medicine, May 4, 1953. †Received for publication July 16, 1953. 
to denote paralysis, spasm, and " neutral" or indeterminate. The muscle which has the greatest agreement or concordance with the same letter is the affected muscle, as will be readily understood from the illustrations.

The Five Diplopia Tests.-These can be made for any distance of fixation, but usually 6 or $7 \mathrm{ft}$. from the patient. The red and green filters are placed before the patient's eyes, over his correcting lenses if necessary, red before the right eye (redright) and green before the left eye. The five tests are always best made in the following order:

(1) Round light directly in front of patient's fixing eye in primary position, to determine which image is higher and which is lower.

(2) With the patient's head held steady, the target is moved to four diagnostic corners (up and left, down and left, up and right, down and right) to determine in what part of the field there is the greatest vertical separation of the images. Right and left refer to the patient's right or left eye throughout.

(3) With the target directly in front of the patient, his head is tilted towards the right and left shoulders to determine where the vertical separation of the images increases.

(4) With the target on the side opposite to that where the greatest vertical separation was found in Test 2, the horizontal relation of the two images is determined (crossed or uncrossed diplopia).

(5) Using the vertical line of light which is also placed on the side opposite to where Test No. 2 showed the greatest vertical separation of the images, we determine which image is tilted and how.

Pascal Muscle Schema.-To understand and apply this method easily one must know the Benzene Ring Muscle Schema (Fig. 1)*, in which each of the six extraocular muscles is placed on one of the six limbs of the ring, each muscle as its name connotes. The complete action of each muscle is shown by the position of the limb (supraduction-infraduction), by the position of the middle dot on the upper and lower limbs (adduction-abduction), and by the slant of the limb (intorsionextorsion). The direction of the greatest up or down action of the vertical muscles is indicated by the position of the upper and lower limbs, which may be considered as "windows" facing in a certain direction.
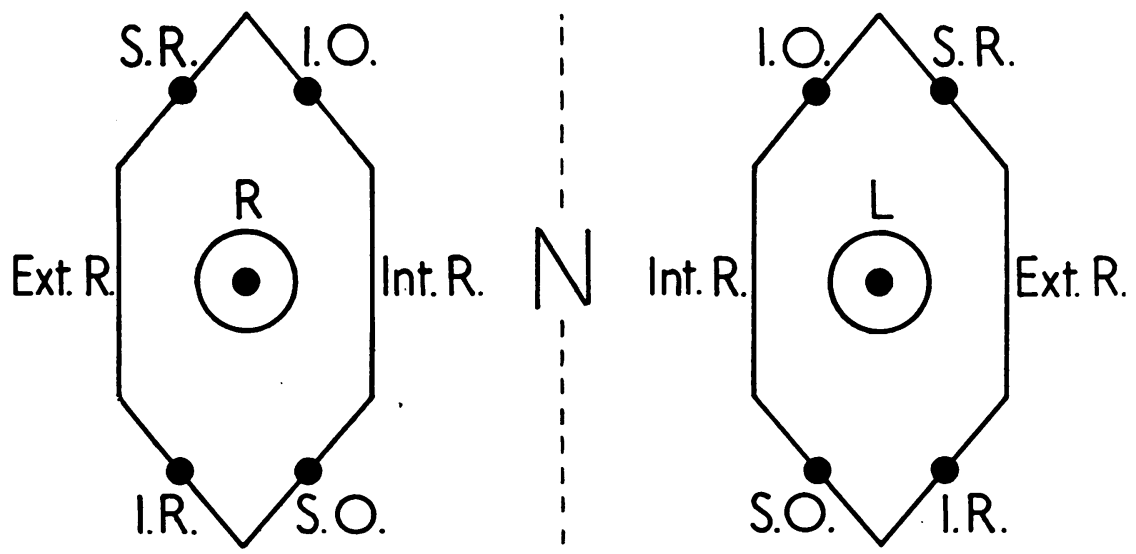

FIG. 1.-Benzene Ring Muscle Schema.

\footnotetext{
* A full discussion of this schema with its sixteen applications is given in Pascal (1952).
} 
Case 1, Tests and Findings.

\section{Application}

(1) Red light lower, green light higher.

(2) Vertical separation of images greatest down and left.

(3) Vertical separation of images increases on tilting head to right shoulder.

(4) Red light to right of green light.

(5) Red vertical line (light) seen tilted, top to (patient's) left.

Interpretation and Tabulation of Findings (Fig. 2):

Test 1.-This shows right eye higher, left eye lower. This may be due to spasm of the right elevators or to paralysis of the right depressors, so we place on line 1, Fig. 2, $\mathrm{S}$ at the right elevators and $\mathrm{P}$ at the right depressors. The condition may also be due to spasm of the left depressors or to paralysis of the left elevators. So we place on same line, $\mathbf{S}$ at the left depressors, and $\mathbf{P}$ at the left elevators.

Test 2.-The muscles which have their greatest vertical action down and left are the right superior oblique and the left inferior rectus (Fig. 2); the limbs are like windows facing in the direction of the field of greatest " down " action of these muscles. Since the vertical diplopia increases in this direction, there may be a paralysis of either of these muscles or a spasm of their direct antagonists. So we place on line 2, P at the right superior oblique and the left inferior rectus, and $S$ at the right inferior oblique and the left superior rectus. The corresponding muscles on the right side of each ring are " neutral " in this movement, so we place a dot $(\cdot)$ at each of these, the right superior rectus, the right inferior rectus, the left inferior oblique, and the left superior oblique.

Test 3.-This shows that any of the muscles which normally produce compensatory levo-torsion may be incapable of doing so (i.e. paralysed), or that their antagonists are spastic. The laevo-torting muscles as seen on the schema are (in the right eye), the right superior rectus and the right inferior oblique, and (in the left eye) the left inferior oblique and the left inferior rectus. So we place on line $3, \mathrm{P}$ at each of these muscles, and $\mathrm{S}$ at their antagonists, that is, at the right inferior oblique, the right inferior rectus, the left superior rectus, and the left superior oblique respectively.

Test 4.-This shows esotropia (homonymous diplopia), which may be caused by spasm of any of the vertical adductors, the vertical recti on the outside of éach ring, or paralysis of any of the vertical abductors, the obliques, on the inside of each ring. So we place on line 4, S at each of the vertical recti and $P$ at each of the obliques.

Test 5.-The tilt of the right-eye image points to paralysis of either muscle in the right eye attached to a limb tilted to the left (the tilt of the false image is the same as the torsion of the paralysed muscle shown by the tilt of the limb in the schema); so we place on line 5, $P$ at the right superior rectus and the right superior oblique. Or the tilt of the image may be due to spasm of the antagonists of the aforementioned muscles; so we place on line 5, $S$ at the right inferior oblique and the right inferior rectus. The muscles in the left eye (which sees the straight-line image) are " neutral", so we place a dot $(\cdot)$ on line 5 at these muscles.

Diagnosis.-We see here an agreement or concordance of the same letter $\mathbf{P}$ (five Ps) at the right superior oblique. We therefore have paralysis of the right superior oblique, left eye fixing.

\section{Case 2, Tests and Findings}

(1) Red light higher green light lower.

(2) Vertical separation of images greatest up and left.

(3) Vertical separation of images increases on tilting head to left shoulder.

(4) Red light seen to left of green light (crossed diplopia).

(5) Red linear line (light) seen tilted, top to (patient's) right. 


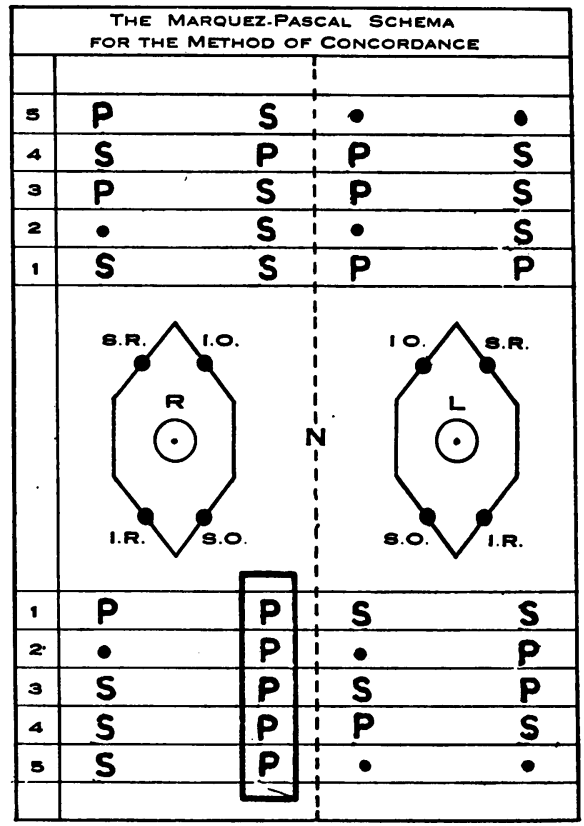

FIG. 2.-Case 1, paralysis of right superior obique, left eye fixing.

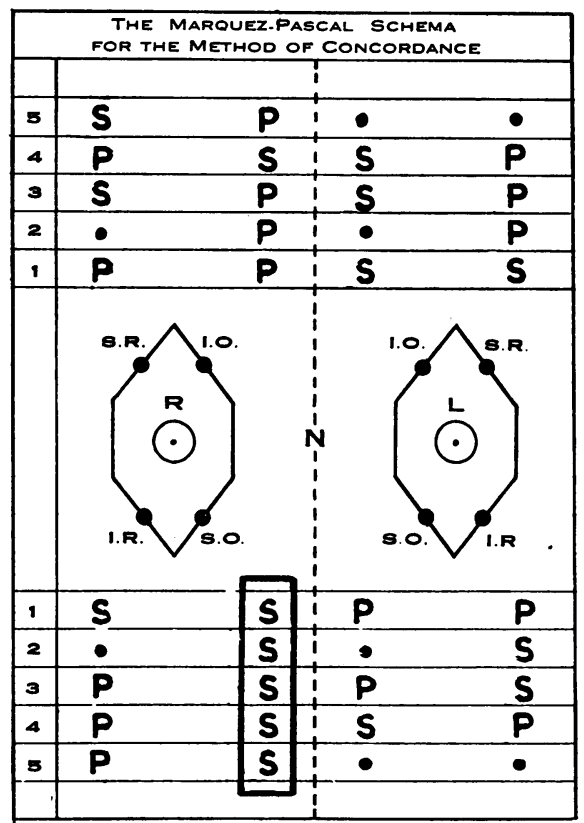

FIG. 3.-Case 2, spasm of right superior oblique, left eye fixing.

\section{Interpretation and Tabulation of Findings (Fig. 3).}

Test 1.-This shows right eye lower. This may be due to paralysis of either elevator of the right eye or to spasm of either depressor of the right eye. So we place on line 1 (Fig. 3), $\mathrm{P}$ at the elevators of the right eye and $\mathrm{S}$ at the depressors of the right eye. Or as the left eye is higher we may have spasm of the left elevators or paralysis of the left depressors. So we place on line $1, \mathrm{~S}$ and $\mathrm{P}$ at these muscles, respectively.

Test 2.-This shows possible paralysis of the muscles whose greatest field of up action is up and left. This points to the right inferior oblique or the left superior rectus (Limbs like windows face up and left to fields of greatest action). So we place on line 2, P at these muscles. Or either of the antagonists of these muscles may be in spasm. So we place on line $2, \mathrm{~S}$ at the right superior oblique and the left inferior rectus. We place a $\operatorname{dot}(\cdot)$ at the muscles on the right side of each ring as they are "neutral" in this movement.

Test 3.-This shows possible paralysis of the dextro-tortors of either eye or spasm of the laevo-tortors of either eye. We therefore place on line $3 \mathrm{P}$ at the dextro-tortors of the right eye, the right inferior oblique and the right inferior rectus, and $\mathbf{S}$ at their antagonists, the right superior rectus and the right superior oblique. We also place $\mathbf{P}$ at the dextrotortors of the left eye, the left superior rectus and the left superior oblique, and $\mathbf{S}$ at their antagonists, the left inferior oblique and the left inferior rectus.

Test 4.-Crossed diplopia shows exotropia. This may be due to spasm of the abductors or to paralysis of the adductors. So we place on line $4 \mathrm{P}$ at the vertical recti of both eyes, and $\mathbf{S}$ at the obliques of both eyes.

Test 5.-This shows torsion of the right eye. Tilt of the image is the same as slant of limb of paralysed muscle. This may be either the right inferior oblique or the right inferior rectus, so we place on line $5 \mathrm{P}$ at the right inferior rectus and the right inferior oblique. Or it may be due to spasm of their torsional antagonists, so we place $S$ at the right superior rectus and the right superior oblique. We place dots $(\cdot)$ at the corresponding muscles on the left ring. 
Diagnosis.-The muscle which shows the greatest degree of concordance is the right superior oblique with five Ss. This shows spasm of the right superior oblique, left eye fixing.

\section{Case 3, Tests and Findings.}

(1) Red light higher green light lower.

(2) Vertical separation of images greatest down and right.

(3) (a) Head tilted to right shoulder, no change in position of lights.

(b) Head tilted to left shoulder, lights move further apart vertically.

(4) Red light to right of green light (uncrossed diplopia).

(5) Green line seen tilted, upper end tilted to (patient's) right.

\section{Interpretation and Tabulation of Findings (Fig. 4).}

Test 1.-This shows right eye is lower and left eye higher. For reasons explained in previous cases we therefore place on line 1 (Fig. 4) $\mathrm{P}$ at right elevators, and $\mathrm{S}$ at right depressors, also $\mathrm{S}$ at left elevators and $\mathrm{P}$ at left depressors.

Test 2.-This direction down and to the right points to the field of greatest (down) action of right inferior rectus and left superior oblique (at "windows" facing that direction). Therefore, we place on line 2, $\mathrm{P}$ at right inferior rectus and left superior oblique, and $\mathbf{S}$ at their vertical antagonists, right superior rectus and left inferior oblique. We place a dot (.) at the muscles on the left, as they are " neutral " in this movement.

Test 3.-This shows that there is weakness or paralysis of the muscles which cause (compensatory) dextro-torsion. We place $\mathbf{P}$ on line 3 at these muscles on both rings, right inferior rectus and right inferior oblique, and left superior rectus and left superior oblique. Or there is spasm of their torsional opponents (i.e. the muscles that produce laevo-torsion), so we place on line $3, \mathrm{~S}$ at right superior oblique and right superior rectus, and left inferior oblique and left inferior rectus.

Test 4.-This shows esotropia (uncrossed diplopia) due either to a spasm of the vertical adductors (vertical recti of both eyes) or to a paralysis of the vertical abductors (obliques of both eyes). We therefore place on line $4, \mathrm{~S}$ at the vertical recti of both eyes and $\mathrm{P}$ at the obliques of both eyes.

Test 5.-The torsion of the false image is the same as the slant of the limb for the paralysed muscle. It really corresponds to the torsion produced by that muscle when normal. Therefore, dextro-torsion of the image of the left eye may be due to paralysis of the dextrotorting muscles in the left eye, left superior rectus or left superior oblique, the limbs which slant to the right. We therefore place on line $5 \mathrm{P}$ at left superior rectus and left superior oblique. Or the condition may be due to spasm of their torsional opponents, left inferior oblique and left inferior rectus, so we place $S$ on line 5 at these muscles. We also place a dot $(\cdot)$ on line 5 at each of the muscles of the right eye as this eye shows no torsion.

Diagnosis.-We see here that the muscle which shows the greatest degree of concordance (concurrence or agreement) of the same symbol $(P)$ is the left superior oblique, with five Ps. We therefore have here a paralysis of the left superior oblique, right eye fixing.

\section{Case 4, Tests and Findings.}

(1) Red light lower than green light.

(2) Vertical separation of images greatest up and left.

(3) Patient does not notice increased vertical separation of images on tilting head to either shoulder.

(4) Red light to left of green light (test made in right side of field).

(5) Green tubular light tilted to right (test made in right side of field). 


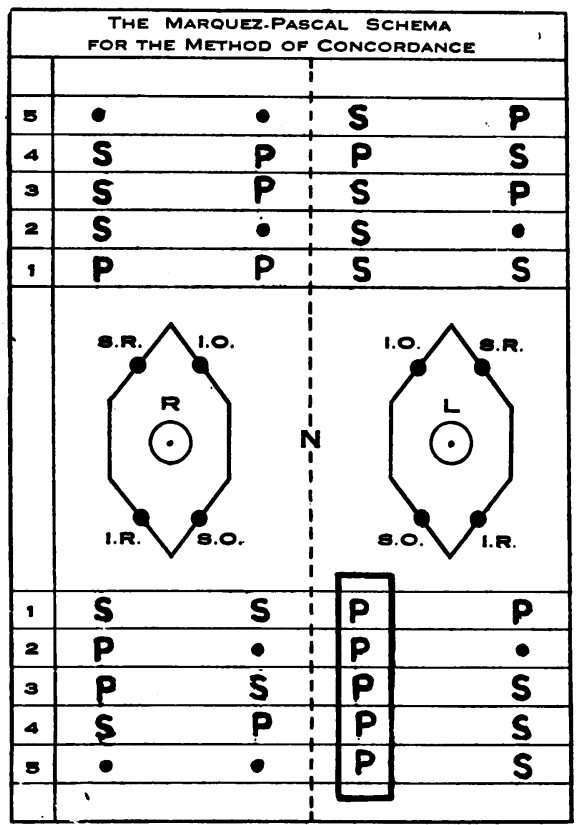

FIG. 4.-Case 3, paralysis of left superior oblique, right eye fixing.

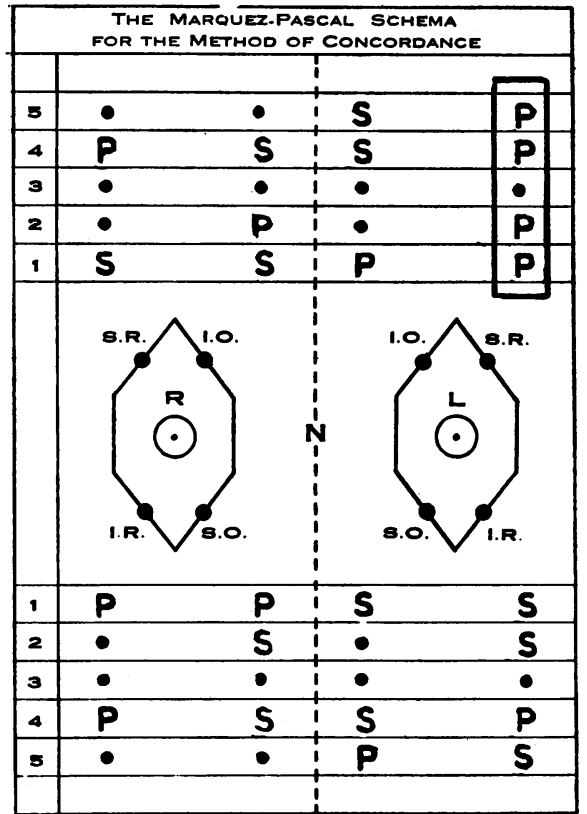

FIG. 5.-Case 4, paralysis of left superior rectus, right eye fixing.

\section{Interpretation and Tabulation of Findings (Fig. 5).}

Test 1.-This shows right eye is higher and left eye lower. We therefore place on line 1 $\mathbf{S}$ at right elevators, and $\mathbf{P}$ at right depressors. We also place $\mathbf{P}$ at left elevators and $\mathbf{S}$ at left depressors.

Test 2.-This direction shows the field of greatest up action of the right inferior oblique and the left superior rectus. The increased vertical separation of the images in that direction may be due to paralysis of the right inferior oblique or the left superior rectus, or to a spasm of their vertical opponents, the right superior oblique and the left inferior rectus. We therefore place on line $2 \mathbf{P}$ at the former and $\mathbf{S}$ at the latter muscles. We place a dot $(\cdot)$ at the muscles on the right side of each ring.

Test 3.-This shows no apparent anomaly of any of the muscles. It may be noted, however, that the effect of tilting the head to either shoulder is more marked in paralysis of an oblique muscle than in paralysis of a rectus muscle. So we place a dot $(\cdot)$ on line 3 , above and below the rings, at all the muscles.

Test 4.- This shows exotropia which may be due to spasm of the vertical abductors or to paralysis of the vertical adductors. So we place on line $4 \mathrm{~S}$ at the obliques and $\mathrm{P}$ at the vertical recti.

Test 5.-Shows torsion (laevo-torsion) of the left eye. The tilt of the image is like the slant of the limb of the paralysed muscle (or opposite to the torsion of the eye). The paralysed muscle may therefore be the left superior rectus of the left superior oblique, and we place on line $5 \mathrm{P}$ at these muscles. Or it may be caused by a spasm of the torsional antagonists of these muscles, so we place $S$ at the left inferior oblique and the left inferior rectus. We place dots $(\cdot)$ at the corresponding muscles on the right ring.

Diagnosis.-The muscle which shows the greatest degree of concordance is the 
left superior rectus with four Ps and a dot. One test was inconclusive, but the other tests show that we have here a paralysis of the left superior rectus, right eye fixing.

\section{Summary}

A method of diagnosing paralytic and spastic strabismus, which is still practically unknown to English-speaking ophthalmologists, is here presented. The method, which is an enlargement and adaptation of a method first presented by Marquez (1942), comprises a co-ordinated system of five diplopia tests, the findings of which are interpreted and tabulated with the aid of the Pascal Muscle Schema. The paretic or spastic muscle is shown by a kind of concurrence or concordance of the same symbol used in the process of tabulation. In actual practice it is not necessary to separate the findings from the interpretation and tabulation on the schema, but this is done here for the purpose of facilitating the exposition. Once the method is understood, all three operations can be carried out in one step.

\section{REFERENCES}

MARqueZ, M. (1942). “Cuestiones Oftalmologicas”. El Colegio de Mexico, Mexico, D.F. (1952). "Oftalmologia Especial Teorica Y Clinica". Prensa Medica Mexicana, Mexico, D.F.

Pascal, J. I. (1952). "Studies in Visual Optics". Mosby, St. Louis.

RIVAS CherIF, M. DE (1949). Arch. Asoc. Evitar Ceguera Mexico, 7, 333.

ScoBeE, R. G. (1952). "The Oculorotary Muscles", Preface, 2nd edition. Mosby, St. Louis. 